\title{
Respuesta a la clara del nogal híbrido y al desmoche del cerezo en plantaciones intensivas del norte de Cáceres, Extremadura, e implicaciones para los sistemas agroforestales
}

\author{
Response to thinning of hybrid walnut and to pollarding of cherry tree \\ in intensive plantations in northern Cáceres, Extremadura, and implications \\ for agroforestry systems
}

\author{
Moreno, G..$^{*}$, Bertomeu, M. ${ }^{1}$, Juárez, E. ${ }^{1}$, Urban, I. ${ }^{2}$, Homar, C.A. ${ }^{2}$ \\ 'Grupo de Investigación Forestal - INDEHESA. Universidad de Extremadura, Plasencia 10600 \\ ${ }^{2}$ Bosques Naturales S.A, Avenida de la Vega 1, 28108, Alcobendas, Madrid \\ *Autor para correspondencia: gmoreno@unex.es
}

\section{Resumen}

En los últimos 20 años se han establecido en España de 8000 a 10000 hectáreas de plantaciones de frondosas nobles, principalmente de nogal y cerezo, para la producción de madera de calidad. Estas plantaciones se han realizado en terrenos agrícolas y se gestionan mayoritariamente de forma intensiva, con podas frecuentes, riego, fertilización y aplicación de herbicidas, con el fin de obtener en unos 25 a 30 años árboles con un diámetro normal de al menos $30 \mathrm{~cm}$. Aunque durante los primeros 15 años el crecimiento del arbolado ha sido el esperado, recientemente se ha observado en plantaciones del norte de Extremadura, una notable reducción del crecimiento anual en diámetro y en altura. Con el fin de promover el crecimiento del arbolado, y de reducir los costes y el impacto ambiental de la gestión intensiva, se están llevando a cabo desde hace varios años estudios sobre los efectos que diversas prácticas agroforestales (pastoreo, cultivos intercalares) tienen sobre el suelo y el arbolado. Se presentan los resultados a los 2 años de iniciar un ensayo de claras en una plantación de nogal híbrido y de desmoche en cerezo para determinar el efecto en el crecimiento diametral del tronco. Tanto los nogales sometidos a la clara como los cerezos desmochados no muestran diferencias significativas en el incremento anual en diámetro con respecto a los árboles control, aunque todavía es pronto para obtener conclusiones. Se discute también la idoneidad de éstas prácticas para las plantaciones de madera de calidad con gestión agroforestal.

\section{Summary}

In the last 20 years, 8000 to 10000 hectares of noble hardwood plantations, mainly walnut and cherry trees, have been established in Spain for the production of quality wood. These plantations have been carried out in agricultural lands and are mostly managed intensively, with frequent pruning, irrigation, fertilization and application of herbicides, in order to obtain trees with a diameter at breast height of at least $30 \mathrm{~cm}$ in about 25 to 30 years. Although during the first 15 years the growth of the trees has been the expected, a notable reduction in annual growth in diameter and height has recently been observed in plantations of northern Extremadura,. In order to promote tree growth, and to reduce the costs and environmental impact of intensive management, studies on the effects that various agroforestry practices (grazing, intercropping) have on the soil and the trees have been carried out for several years. The results are presented after 2 years of starting a thinning trial in a plantation of hybrid walnut and a pollarding trial of cherry trees to determine the effect on stem diameter growth. Both the walnut trees subjected to thinning and the pollarded cherry trees do not show significant differences in annual diameter increment with respect to the control trees, although it is still early to draw conclusions. The suitability of these practices for quality wood plantations with agroforestry management is also discussed.

Palabras clave: agroforestería, cavitación, embolismo, madera de calidad, trasmocho.

Keywords: agroforestry, cavitation, embolism, wood quality, pollarding 


\section{Introducción}

En las dos últimas décadas, la escasez de la madera de calidad y su demanda en el mercado han impulsado en España el establecimiento de un importante número de plantaciones con especies de madera nobles. Con Castilla y León a la cabeza (2338 ha), seguida de Extremadura (1457 ha) y Castilla-La Mancha (1311 ha), además de varios cientos de hectáreas en Galicia, Andalucía, Navarra, Valencia, Cataluña y Aragón, se estima que existen actualmente en España entre 8000 a 10000 ha de frondosas nobles, principalmente de nogal (Juglans sp.) y cerezo (Prunus avium L.) (Aletà y Vilanova, 2014).

Estas plantaciones se han realizado mayormente en terrenos agrícolas de buena calidad (terrenos llanos o con pendiente leve y suelos profundos) y se gestionan siguiendo prácticas comunes en la agricultura y la silvicultura intensiva: densidades bajas de plantación (entre 100 a 600 árboles ha ${ }^{-1}$ ); podas frecuentes; riego, fertilización y aplicación de herbicidas para promover el rápido crecimiento y acortar así los turnos a 25 ó 30 años (Aletà y Vilanova, 2014). Así en los primeros 10-15 años se han observado incrementos medios anuales en diámetro de $1 \mathrm{~cm}$, llegando con cierta frecuencia hasta los $2 \mathrm{~cm}$, y un crecimiento medio anual en altura de hasta $1 \mathrm{~m}$ (Aletà et al., 2003; Aletà y Vilanova, 2006; Urbán et al., 2013).

Sin embargo en los últimos años se ha venido observando en plantaciones del norte de Cáceres (Extremadura) una reducción del incremento anual en diámetro, que unido al alto coste económico y ambiental de la gestión intensiva ponen en riesgo el éxito de estas plantaciones. Las causas de esta reducción en el crecimiento diametral difieren según la especie. En las plantaciones con nogal híbrido es probablemente debido a la competencia intraespecífica que, a pesar de que la densidad inicial de plantación fue baja (333 árboles ha ${ }^{-1}$ ), ocurre una vez que los árboles ocupan el espacio disponible a su alrededor. En las plantaciones con cerezo, los árboles muestran síntomas de cavitación y embolismo severo. Estos problemas apuntan a la necesidad de probar nuevas prácticas de manejo del suelo y del arbolado que favorezcan el crecimiento de éste y que a la vez ayuden a reducir los costes de gestión y mantenimiento de la plantación.

Las prácticas agroforestales pueden mejorar sensiblemente la gestión de las plantaciones de frondosas nobles. Las plantación entre árboles de especies de acompañamiento, la mejora genética tradicional (selección de árboles plus y desarrollo de nuevos híbridos y clones) y adecuadas podas de formación, pueden mejorar notablemente la rentabilidad de las plantaciones al conseguirse madera de calidad, con fustes más rectos y cilíndricos y con menos ramas, y generar ingresos anuales (Aletà et al., 2003; Coello y Piqué, 2009; Urbán et al., 2013). El uso de acolchados en las primeras edades de la plantación junto con el pastoreo mejoran el crecimiento del arbolado al controlar la competencia de la vegetación herbácea y mejorar el suelo, y pueden reducir completamente el uso de herbicidas o del laboreo con la consiguiente reducción en los costes de mantenimiento. Efectos similares se pueden obtener también con la implantación intercalada de leguminosas o de cultivos anuales (como el cereal), ya que pueden generar ingresos intermedios, mejorar la nutrición nitrogenada del 
arbolado y reducir el uso de fertilizante y herbicidas (López-Díaz et al., 2013; Coello y Piqué, 2016).

La clara y el desmoche son prácticas de gestión del arbolado que pueden también favorecer el crecimiento del arbolado. El aclarado es una práctica silvícola muy común, tanto en bosques como en plantaciones, que persigue promover el crecimiento de los mejores árboles al liberarlos de la competencia de los pies vecinos (Assmann, 1970; Nyland, 2002).

El desmoche fue una práctica muy extendida en Europa en el pasado, tanto en masas forestales como en sistemas agroforestales y silvopastorales. Consiste en la eliminación de las ramas superiores del árbol, promoviendo un denso rebrote y follaje a la altura del corte (Cantero et al., 2008; Ruano, 2013). Tradicionalmente se realizaba para conseguir leña y ramón para la alimentación animal. Por ejemplo, el fresno (Fraxinus angustifolia) ha sido muy utilizado como árbol forrajero en muchas regiones europeas y en el centro y el oeste ibérico. El desmoche también se ha realizado para reducir el sombreado de los cultivos intercalados; de hecho, frecuentemente el desmoche se anticipaba al año de cultivo. Finalmente en la fruticultura, el desmoche se ha practicado como mecanismo de saneamiento de los árboles que presentaban ramas senescentes y como mecanismo de control de la altura de recolección de fruta. La observación de grandes diámetros $(\mathrm{dbh}>1 \mathrm{~m})$ que alcanzan aquellos árboles que fueron periódicamente desmochados durante décadas (o siglos) nos hace pensar que éste podría ser una buena práctica silvícola que permita seguir creciendo en diámetro a los cerezos (y otras frondosas) cultivados para producir madera de calidad. De hecho en un estudio reciente Ghahramany et al. (2016) concluyen que el desmoche, practicado aún hoy día por los agricultores del oeste de Irán para la obtención de forraje y leñas, tiene un impacto positivo en el incremento diametral del roble del Líbano (Quercus libani Oliv.). El desmoche podría beneficiar al árbol en la medida en que mitiga el déficit hídrico al reducir la superficie transpiratoria del mismo, y al eliminar las ramas más distales a las que el agua llega con más dificultad. En las plantaciones de cerezo de madera noble el objetivo sería conseguir los diámetros mayores en los primeros $3 \mathrm{~m}$ de altura de tronco, el mínimo exigido por los aserraderos.

En este trabajo presentamos los resultados iniciales de dos experiencias llevadas a cabo en dos plantaciones en el norte de Cáceres, Extremadura, para determinar el efecto en el crecimiento diametral del arbolado de una clara de nogal híbrido y el desmoche de cerezos. Las hipótesis de trabajo son las siguientes:

1.- En las plantaciones intensivas de nogal híbrido con baja densidad inicial (de menos de 400 pies ha $^{-1}$ ), para poder mantener un incremento anual medio en diámetro de $1 \mathrm{~cm}$ es necesario realizar a la edad correspondiente a la mitad del turno una clara de, al menos, entre $40-50 \%$ de peso.

2.- En las plantaciones intensivas de cerezo, el incremento anual en diámetro de la troza basal (hasta los 3 metros) de los árboles afectados por embolismo y cavitación que se desmochan (eliminándose el tronco y las ramas muertas de la parte superior) es similar al de los árboles sin desmochar. 
Los ensayos establecidos para refutar estas hipótesis son parte de un programa más amplio de investigación entre la Universidad de Extremadura y la compañía Bosques Naturales con el objeto de mejorar el rendimiento económico y ambiental de las plantaciones de madera de calidad a través de las prácticas agroforestales, que incluyen el manejo silvopastoral con ovejas, la mejora de pastos con siembra de leguminosas y los cultivos intercalares.

\section{Materiales y métodos}

\subsection{Ensayo de Aclarado: Plantación de nogal}

En Enero de 2014 se inició un ensayo en una plantación de nogales híbridos ( $\mathrm{Ju}$ glans major $x$ J. regia, progenie comercial de 'Mj209xRa') para determinar el efecto del aclarado en el incremento radial de los troncos. El estudio se realizó en una plantación de la compañía Bosques Naturales en Madrigal de la Vera (Cáceres; ETRS89 huso 20: X: 298303 Y: 4442 326). La plantación se hizo en el año 2000, con un marco de $5 \times 6 \mathrm{~m}$, sobre un suelo aluvial arenoso con abundantes gravas. La plantación ha sido manejada hasta el año 2011 mediante la aplicación de herbicidas en la línea de árbol y gradeo anual de las calles. Desde el 2011 hasta la actualidad es manejada mediante pastoreo con ovejas en sustitución del herbicida y el gradeo.

Para el ensayo se eliminaron más de 100 árboles (los que presentaban más imperfecciones en la forma del tronco), sin seguir ningún esquema predefinido (clara selectiva). De este modo a los árboles de futuro (los que quedan en pie) se les liberó de la competencia de hasta 5 árboles vecinos (de un máximo de 8 árboles vecinos). Como resultado se cuenta con un gradiente de intensidad de aclarado, cuantificado en base al número y tamaño de los árboles vecinos eliminados, teniendo en cuenta la distancia de los árboles eliminados respecto a los árboles de futuro.

Intensidad de Aclarado $=\Sigma \mathrm{DBH}(\mathrm{cm}) \operatorname{distancia}(\mathrm{m})^{-1}$;

(incluye hasta un máximo de 8 árboles alrededor de los árboles de futuro).

En Enero de 2015 y 2016, el diámetro normal del tronco de los árboles restantes fue medido.

\subsection{Ensayo de Trasmocho: Plantación de cerezo}

El ensayo sobre el desmoche comenzó en Enero de 2015 en una plantación de cerezos de la compañía Bosques Naturales en Madrigal de la Vera (Cáceres), para determinar si los árboles afectados por una severa cavitación en sus ramas superiores experimentaban alguna mejoría significativa en el crecimiento del tronco tras el desmoche.

Los cerezos del ensayo (Prunus avium L. clon 'Eurocherry C-9') fueron plantados en 2004 en un marco de 5x5 m. Durante los primeros 3 años fueron podados en invierno con objeto de conformar un tronco único vertical. Después los árboles fueron podados anualmente en primavera hasta 2011 para eliminar las ramas indeseadas. 
Desde 2011 sólo los brotes epicórmicos han sido eliminados. Como prácticas culturales se aplican herbicidas en la línea de los árboles y las calles entre líneas se gradean anualmente.

El experimento incluye 5 pares de parcelas seleccionadas al azar dentro de la plantación. Cada parcela incluye 3 filas de árboles de 6 árboles cada una (18 árboles por parcela). En cada par de parcelas, una de ella fue asignada al tratamiento de desmoche y la otra sirvió de control. En total 90 árboles (5 parcelas x 18 árboles) fueron desmochados.

En otoño de 2014, antes de la caída de las hojas, los árboles fueron fotografiados individualmente para identificar más tarde en invierno, en el momento del desmoche, la porción del tronco con ramas vivas y aparentemente no afectadas por la cavitación de sus vasos. En Febrero de 2015 los árboles fueron desmochados guiados por las fotografías tomadas en el otoño anterior. En el momento del desmoche, se midió la altura del árbol y el diámetro normal del tronco. La longitud de la troza desmochada y el diámetro en la base de la sección del corte también fueron medidas (Tab. 1).

Tabla 1. Valores medios ( \pm D.E.) iniciales del diámetro normal (Dap), altura total $(H)$ y de la longitud y el diámetro en la base (Dbase) de la troza cortada de los cerezos en el ensayo de desmoche, mostrados por parcelas ( $\mathrm{n}=18$ árboles por parcela).

\begin{tabular}{|c|c|c|c|c|c|c|c|}
\hline \multicolumn{3}{|c|}{ CONTROL } & \multicolumn{5}{c|}{ DESMOCHE } \\
\hline Parcela & Dap (cm) & H $(\mathrm{m})$ & Parcela & Dap (cm) & H (m) & Dbase (cm) & Longitud (m) \\
P1 & $17.5 \pm 2.1$ & $6.2 \pm 1.0$ & P2 & $18.6 \pm 1.6$ & $8.1 \pm 0.5$ & $9.1 \pm 1.2$ & $4.7 \pm 0.6$ \\
P4 & $18.7 \pm 2.4$ & $7.6 \pm 0.8$ & P3 & $18.8 \pm 1.5$ & $8.1 \pm 0.5$ & $8.3 \pm 1.0$ & $4.3 \pm 0.5$ \\
P5 & $19.7 \pm 1.1$ & $8.0 \pm 0.9$ & P6 & $19.4 \pm 1.5$ & $8.0 \pm 0.6$ & $8.5 \pm 0.8$ & $4.3 \pm 0.5$ \\
P7 & $17.0 \pm 1.4$ & $6.9 \pm 0.7$ & P8 & $15.7 \pm 1.3$ & $7.2 \pm 0.5$ & $8.3 \pm 0.6$ & $4.5 \pm 0.4$ \\
P9 & $19.6 \pm 1.4$ & $6.1 \pm 0.4$ & P10 & $18.2 \pm 1.9$ & $7.3 \pm 0.6$ & $8.5 \pm 0.7$ & $3.8 \pm 0.4$ \\
\hline
\end{tabular}

\section{Resultados}

Los resultados del experimento de la clara en nogal mostraron un ligero efecto positivo de la reducción de la competencia sobre el crecimiento acumulado de 2 años del diámetro normal de los árboles $(\mathrm{r}=0.25 ; \mathrm{p}<0.001$; Fig. 1). No obstante, de momento la respuesta es más débil de lo esperado, y las mediciones se seguirán realizando partiendo de la hipótesis de que los árboles restantes necesitan más tiempo para colonizar el espacio, especialmente el suelo.

Respecto al ensayo de desmoche los resultados del primer año (incremento anual del diámetro normal del tronco) no mostraron diferencias significativas entre las 2 categorías de árboles, los desmochados y los árboles control $(\mathrm{F} 1,168=0.132 ; \mathrm{p}=0.72)$, con un crecimiento escaso en ambos grupos de árboles ( $0.35 \pm 0.01$ S.E. vs $0.37 \pm 0.05$ S.E., respectivamente; Tab. 2). Se hará un seguimiento de los árboles durante los pró- 


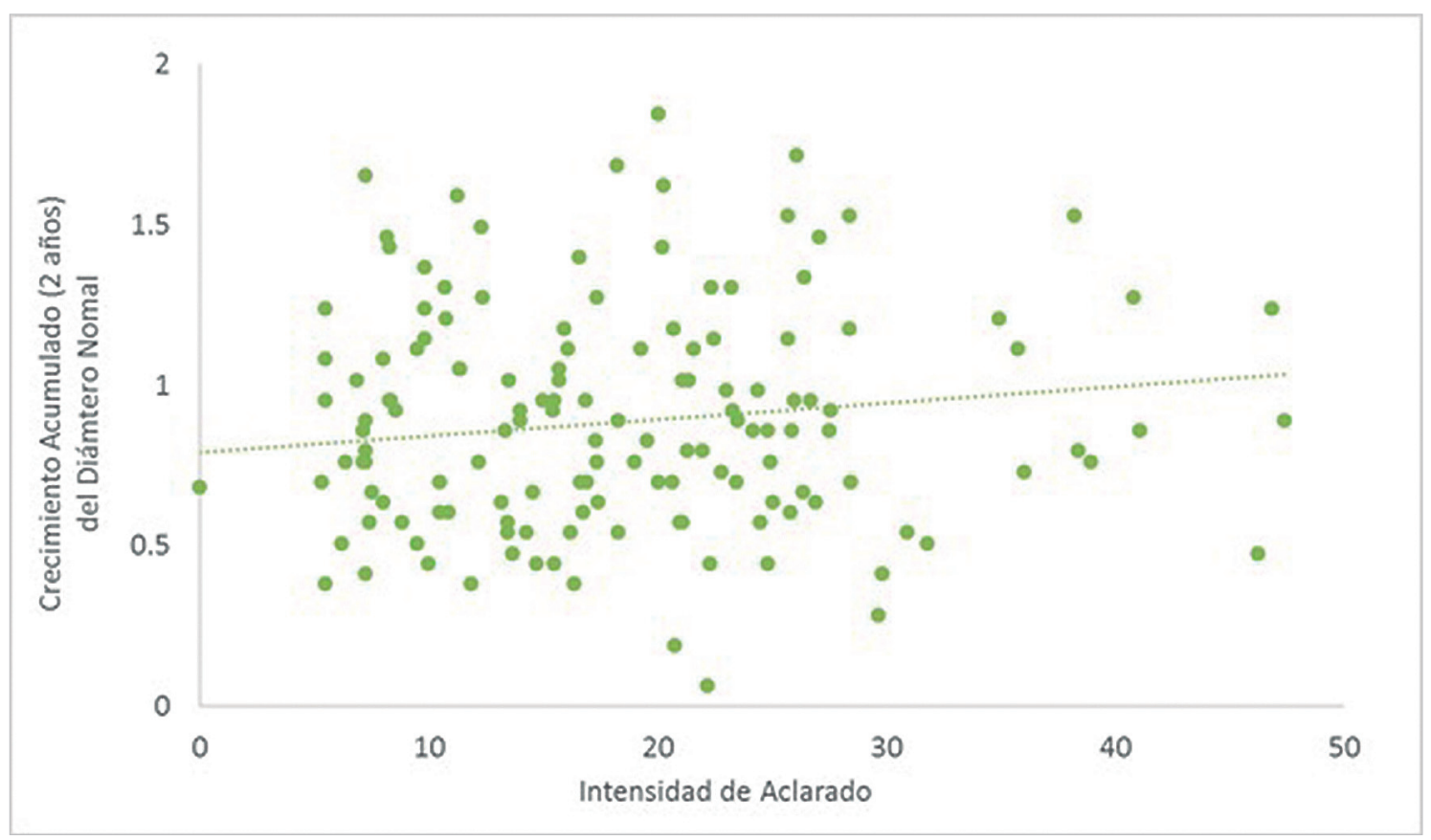

Figura 1. Respuesta del arbolado a la intensidad del aclarado (adimensional; ver definición en sección de métodos). Se muestran los datos del crecimiento acumulado de 2 años $(\mathrm{cm})$ en el diámetro normal de los tron$\cos . \mathrm{R}^{2}=0.0147 ; \mathrm{p}$ (intercepción $)<0.0001 ; \mathrm{p}($ Intensidad de Aclarado $)=0.00015$.

ximos 2 años para comprobar si la mejora fisiológica esperada en los árboles desmochados conlleva a medio plazo una mejora en el crecimiento radial de los troncos.

Tabla 2. Incremento anual del diámetro del tronco $\left(\mathrm{DBH} ; \mathrm{cm}\right.$ año $\left.{ }^{-1}\right)$ de los árboles desmochados frente a los árboles control.

\begin{tabular}{|c|c|c|c|}
\hline Tratamiento & $\begin{array}{c}\text { Incremento DBH } \\
\text { cm añ }^{-1}\end{array}$ & Error estándar & N \\
\hline Control & 0.369 & 0.052 & 90 \\
Desmoche & 0.350 & 0.015 & 90 \\
\hline
\end{tabular}

\section{Discusión}

Aunque para poder refutar o validar las hipótesis de este estudio es necesario hacer un seguimiento de los ensayos durante al menos 2 años más, desarrollamos en este apartado algunos argumentos sobre la idoneidad de la clara y el desmoche como prácticas de manejo del arbolado en plantaciones de madera de calidad con gestión agroforestal.

El impacto positivo de los distintos tipos de claras sobre la dinámica del rodal y el crecimiento de los árboles de futuro en distintos tipos de masas forestales (desde bosques naturales o semi-naturales hasta plantaciones tanto monoespecíficas como 
mixtas) está avalado por varios siglos de evidencia empírica y desde hace algo más de 100 años por numerosos estudios científicos (Puettmann et al., 2009; Pretzch, 2009). Es de esperar que las plantaciones de maderas nobles con gestión intensiva no sean una excepción y que por tanto la clara sea una práctica necesaria tan pronto como el arbolado comience a competir por los recursos luz, agua y nutrientes si se desea mantener un crecimiento adecuado del arbolado. La débil respuesta inicial a la clara de los árboles de futuro en nuestro ensayo nos induce a pensar si el nogal híbrido necesita más tiempo para responder a la clara y seguir creciendo a un ritmo similar al de los primeros años. Sin embargo en plantaciones con gestión intensiva (con riego y fertilización), como en la de este experimento, se espera una mejor y más rápida respuesta del arbolado a la clara ya que los recursos bajo el suelo no son limitantes (Ong et al., 1996; Berlyn and Cho, 2000). Cabe por tanto plantearse si se debería haber realizado la clara a una edad más temprana. En este sentido Coello et al. (2013) recomiendan para una plantación pura de nogal hibrido de 18 años de edad, $20 \mathrm{~cm}$ de diámetro medio y plantada a 9x6 $\mathrm{m}\left(185\right.$ pies ha $\left.^{-1}\right)$ la realización de una clara con un $25 \%$ de peso (eliminación de 45 árboles ha ${ }^{-1}$ ). Esto sugiere que en nuestro caso la clara debería haber sido más temprana, ya que se realizó en una plantación con 14 años de edad y casi el doble de densidad (333 pies ha $\left.{ }^{-1}\right)$. Además el temperamento intolerante del nogal, o con una sensibilidad media a la competencia por luz (Coello et al., 2013), refuerza la hipótesis de la idoneidad de una clara más temprana.

La clara es una práctica adecuada no sólo para las plantaciones de maderas nobles con gestión puramente selvícola sino también para aquellas con gestión agroforestal, ya que en éstas favorece también el crecimiento de los cultivos intercalares o del estrato herbáceo. En plantaciones de pinos, por ejemplo, sólo existe pasto suficiente para el ganado durante los primeros años de establecimiento y desarrollo del arbolado y después de realizar una clara (Beckerman, 2000). En plantaciones agroforestales con riego y fertilización debería haber en general una mejor respuesta a la clara no sólo de los árboles sino también de los cultivos intercalares ya que los recursos bajo el suelo no son limitantes. Por tanto a la hora de decidir el espaciamiento inicial de la plantación se deberá tener en cuenta el tipo de gestión (puramente selvícola o agroforestal), así como la disponibilidad de agua, de nutrientes y de luz a medida que el espaciamiento aumenta con las claras (Berlyn and Cho, 2000).

Respecto al desmoche, no existen prácticamente experiencias respecto a su idoneidad como técnica de gestión del arbolado en plantaciones de madera de calidad. Cisneros et al. (2006) sugieren que para el fresno (Fraxinus angustifolia), si se eleva la altura de corte por encima de los 2.5-3 m de altura, el desmoche permitiría compatibilizar la producción de madera de calidad con leñas y ramón para el ganado. En sistemas agroforestales con cultivos intercalados, el desmoche, al igual que las claras y podas, beneficiaría a los cultivos anuales al reducir el nivel de sombra. Sin embargo, aunque los grandes diámetros que presentan los árboles que han sido durante años desmochados (hayas, fresnos, robles y otras especies) nos hagan pensar en la compatibilidad de esta práctica con la producción de trozas con diámetro comercial, también parece lógico pensar que tras el desmoche el árbol utilizara la mayor parte de los recursos disponibles en la producción de ramas y follaje, en detrimento del cre- 
cimiento diametral del tronco. En un estudio sobre un sistema agroforestal con Leucaena en mezcla con cultivo de sorgo, Hocking y Rao (1990) concluyen que el desmoche de Leucaena en el momento de la siembra del sorgo fue el sistema de gestión más productivo de biomasa foliar, leña y sorgo, pero resultó en menor biomasa en tronco de Leucaena. Por el contrario, un estudio reciente de un sistema silvopastoral tradicional en Zagros, Irán, sobre el impacto del desmoche en el crecimiento diametral del roble del Líbano (Quercus libani Oliv.) concluye que el desmoche tiene un efecto positivo en el incremento diametral, siendo las diferencias estadísticamente significativas en el caso de los árboles de las clases de edad superiores (Ghahramany et $a l ., 2016)$. Para la producción de maderas nobles, la cuestión por tanto reside en si el desmoche es compatible con la producción de madera con diámetro comercial en un tiempo razonable y asumible por el gestor.

Los resultados del estudio sobre el desmoche en cerezo aquí presentado no nos permiten sacar ninguna conclusión sobre el efecto de ésta práctica en el crecimiento del árbol. Como se indicó en la introducción, los árboles de la plantación presentaban síntomas de cavitación y embolismo y como resultado el incremento anual en diámetro y en altura se había reducido notablemente en los últimos años. Las especies forestales difieren considerablemente en su vulnerabilidad a la cavitación. Las menos vulnerables son normalmente las más tolerantes a la desecación (Lambers et al., 2008). Por ejemplo, entre las especies peninsulares de Quercus, Q. coccifera es la menos vulnerable a la cavitación inducida por el déficit hídrico, mientras que $Q$. robur y $Q$. pyrenaica son mucho más vulnerables (Vilagrosa et al., 2012). También ha sido documentada la diferencia fenotípica en la vulnerabilidad a la cavitación. Las ramas de haya (Fagus sylvatica) expuestas al sol son menos vulnerables a la cavitación que las ramas desarrolladas a la sombra (Cochard et al., 1999). Dentro de la misma planta, la vulnerabilidad también difiere entre órganos, siendo las raíces más sensibles que los tejidos leñosos aéreos (Vilagrosa et al., 2012). Un estudio reciente mostró que $P$. avium era uno de las especies de Prunus más vulnerables a la cavitación (la tercera de diez; Cochard et al., 2008). El cerezo además es muy sensible a una variedad de plagas y enfermedades y exigente en cuanto a las condiciones de sitio (suelos profundos, textura adecuada, no encharcables, sensible a las altas temperaturas) (Coello et al., 2013). Por todo ello, en los últimos años han surgido serias dudas sobre la idoneidad de $P$. avium para producción de madera de calidad en condiciones mediterráneas, incluso en los casos en que los árboles dispongan de abundante riego y fertilización.

\section{Conclusiones}

A los dos años del inicio de los ensayos de claras en una plantación de nogal y de los de desmoche en cerezo no se observan diferencias significativas en el crecimiento diametral entre árboles tratados y los que no lo han sido. Se precisa realizar un seguimiento en ambos ensayos durante los próximos años para determinar si estas prácticas favorecen el crecimiento diametral de los árboles y son adecuadas para el 
manejo del arbolado en plantaciones de maderas nobles con gestión puramente silvícola o agroforestal.

\section{Bibliografía}

Aletà, N., Ninot, A., Voltas, J., 2003. Caracterización del comportamiento agroforestal de doce genotipos de nogal (Juglans sp.) en dos localidades de Cataluña. Invest. Agrar: Sist. Recur. For. 12 (1), 39-50.

Aletà, N., Vilanova, A., 2006. El Nogal Híbrido. Navarra Forestal 13, 18-21.

Aletà, N., Vilanova, A., 2014. Les plantacions espanyoles de fusta d'alt valor. Catalunya Forestal 119, 21-24.

Assmann, E., 1970. The principles of forest yield study. Pergamon, Oxford, New York.

Beckerman A.P., 2000. Some Applications of Multitrophic Level Ecological Theory to Agroforestry Systems. In: Ashton S.M., Montagnini, F. (eds.) The silvicultural basis for agroforesty systems. CRC Press, Florida, pp. 84-117.

Berlyn, G.P., Cho, J., 2000. Light, Moisture, and Nutrient Use by Plants. In: Ashton S.M., Montagnini, F. (eds.) The silvicultural basis for agroforesty systems. CRC Press, Florida, pp. 16-46.

Cantero, A., Passola, G., Aragón, A., de Francisco, M., Mugarza, V., Ria-o, P. 2008. Apuntes sobre Trasmochos. Guía de Buenas Prácticas para el Trasmocheo. http://www.trasmochos.net/images/Trasmochos/Documentos/apuntes_sobre_trasmochos.pdf. Accessed. 12 December 2015.

Cisneros, O., Martínez, V., Montero, G., Alonso, R., Turrientes, A., Ligos, J., Santana, J., Llorente, R., Vaquero, E., 2006. Plantaciones de Frondosas en Castilla y León. Cuaderno de Campo, http://bibliotecadigital.jcyl.es/i18n/consulta/registro.cmd?id=22596 Accessed. 22 June 2016.

Cochard, H., Barigah, S.T., Kleinhentz, M., Eshel, A., 2008. Is xylem cavitation resistance a relevant criterion for screening drought resistance among Prunus species? J Plant Physiol 165, 976-982. https://doi.org/10.1016/j.jplph.2007.07.020

Coello, J., Becquey, J., Gonin, P., Ortisset., J.P., Desombre, V., Baiges, T., Piqué, M., 2013. Frondosas productoras de madera de calidad: ecología y selvicultura de especies para el ámbito pirenaico y regiones limitrofes. Centro de la Propiedad Forestal. Generalitat de Cataluña.

Coello, J., Piqué, M., 2009. Plantaciones mixtas de nogal, serbal y fresno para la producción de madera de calidad y restauración forestal. http://secforestales.org/publicaciones/index. php/congresos_forestales/article/view/16766/16609 Accessed. 5 September 2016

Coello, J., Piqué M., 2016. Acondicionadores y cubiertas del suelo para una plantación de árboles más eficiente y sostenible. Centro Tecnológico Forestal de Cataluña, Solsona.

Ghahramany, L., Shakeri, Z., Ghalavand, E., Ghazanfari, H., 2016. Does diameter increment of Lebanon oak trees (Quercus libani Oliv.) affected by pollarding in Northern Zagros, Iran? Agroforest. Syst. 91(4), 741-748. https://doi.org/10.1007/s10457-016-9944-9

Hocking, D., Rao, D.G., 1990. Canopy management possibilities for arboreal Leucaena in mixed sorghum and livestock small farm production systems in semi-arid India. Agroforest. Syst. 10, 135-152. https://doi.org/10.1007/BF00115362 
Lambers, H., Chapin III F.S., Pons T.J., 2008. Plant Physiological Ecology. Springer Science + Business Media, New York. https://doi.org/10.1007/978-0-387-78341-3

López-Díaz M.L., Moreno, G., Bertomeu, M., 2013. Manejo silvopastoral para la producción de madera de calidad: bases funcionales, productividad y servicios ambientales. http:// www.congresoforestal.es/index.php?men=405\&idCP=81 Accessed. 18 May 2016

Nyland, R., 2002. Silviculture: Concepts and Applications. McGraw-Hill, New York.

Ong, C.K., Black, C.R., Marshall, F.M., Corlett, J.E. 1996. Principles of Resource Capture and Utilization of Light and Water. In: Ong, C.K. and Huxley, P. (eds.): Tree-Crop Interactions: a Physiological Approach. Wallingford, UK: CAB International, pp. 73-158.

Pretzsch, H., 2009. Forest Dynamics, Growth and Yield. From Measurement to Model. Springer-Verlag, Berlín.

Puettmann, K.J., Coates, C., Messier, C., 2009. A critique of silviculture: managing for complexity. Island Press, Washintong D.C.

Ruano, A., 2013. Guided Pollards and the Basque Woodland During the Early Modern Age. In: Rotherham I.D. (ed.), Cultural Severance and the Environment, Environmental History 2. Springer, pp. 147-160. https://doi.org/10.1007/978-94-007-6159-9_10

Urbán, I., Licea, R., González, C., Villar de Arcos, F., Santaclara, O., Merlo, E., 2013. Characterization of walnut hybrid plus trees for good quality wood plantations. International Scientific Conference on Hardwood Processing (ISCHP13). https://www.researchga te.net/publication/264917078_Characterization_of_walnut_hybrid_plus_trees_for_good _quality_wood_plantations Accessed. 5 September 2016

Vilagrosa, A., Chirino, E., Peguero-Pina, J.J., Barigah T.S., Cochard, H., Gil-Pelegrín, E., 2012. Xylem Cavitation and Embolism in Plants Living in Water-Limited Ecosystems. In: Aroca, R. (ed.) Plant Responses to Drought Stress, Springer-Verlag Berlin, pp. 63109. https://doi.org/10.1007/978-3-642-32653-0_3 